\title{
Microencapsulação de pigmento natural de urucum e avaliação de sua estabilidade
}

\author{
Microencapsulation of annatto and stability evaluation's \\ Microencapsulación de pigmento de urúcum natural y evaluación de su estabilidad
}

Recebido: 12/07/2021 | Revisado: 21/07/2021 | Aceito: 25/07/2021 | Publicado: 01/08/2021

\author{
Patrícia Beltrão Lessa Constant \\ ORCID: https://orcid.org/0000-0001-7095-940X \\ Universidade Federal de Sergipe, Brasil \\ E-mail: pblconstant@yahoo.com.br \\ Andréa Gomes da Silva \\ ORCID: https://orcid.org/0000-0002-8956-0121 \\ Universidade Estadual do Sudoeste da Bahia, Brasil \\ E-mail: gomesa28@gmail.com \\ Ângela da Silva Borges \\ ORCID: https://orcid.org/0000-0003-1278-9638 \\ Universidade Federal de Sergipe, Brasil \\ E-mail: angelasborges@yahoo.com.br \\ Filipe Oliveira Melo \\ ORCID: https://orcid.org/0000-0002-4702-1162 \\ Universidade Federal de Sergipe, Brasil \\ E-mail: filipe.melo@ifal.edu.br \\ Flávia Escapini Fanchiotti \\ ORCID: https://orcid.org/0000-0002-1486-2846 \\ Universidade Federal de Pernambuco, Brasil \\ E-mail: flavia.fanchiotti@ufpe.br \\ Flávia Luiza Araújo Tavares da Silva \\ ORCID: https://orcid.org/0000-0001-7756-9252 \\ Universidade Federal de Sergipe, Brasil \\ E-mail: flavialuats@hotmail.com \\ Paulo Cesar Stringheta \\ ORCID: https://orcid.org/0000-0002-1229-7038 \\ Universidade Federal de Viçosa, Brasil \\ E-mail: pstringheta@gmail.com
}

\begin{abstract}
Resumo
A microencapsulação é um processo que tem como base o confinamento da espécie ativa dentro de uma cápsula microscópica, que lhe pode conferir proteção contra fatores lábeis. Tal técnica vem sendo utilizada na indústria de flavorizantes e apresenta grande potencial na solução de dificuldades relativas ao uso de corantes naturais. O presente trabalho foi realizado visando aplicar a técnica de microencapsulação em corantes naturais para o aumento de sua estabilidade e para a melhoria na sua utilização como ingrediente alimentício. Empregou-se o processo de encapsulação por "spray drying". O corante escolhido foi a bixina, que é a fração lipossolúvel do pigmento do urucum (Bixa orellana, L), e os agentes encapsulantes foram maltodextrina, goma-arábica e beta-ciclodextrina. A partir destes três agentes encapsulantes obtiveram-se várias formulações, usando cada agente separadamente ou misturados. Em seguida, foram feitos testes de estabilidade para os produtos microencapsulados e para o norbixinato usado como testemunha para avaliação do efeito da luz. Os produtos microencapsulados mostraram-se mais estáveis que o pigmento sem microencapsulação e, entre estes, aqueles compostos por formulações de misturas de goma-arábica e maltodextrina tiveram de forma geral, os melhores desempenhos. A beta-ciclodextrina, por sua vez, mostrou ser um agente encapsulante bastante eficiente, e, apesar do alto custo, aparece como uma opção viável no que diz respeito ao aumento da estabilidade de pigmentos naturais.
\end{abstract}

Palavras-chave: Agentes encapsulantes; Bixina; Colorimetria; Corantes alimentícios; Microcápsulas.

\begin{abstract}
Microencapsulation is a process based on confining the active species (colorant) inside a microscopic capsule, which can confer it protection against labile factors such as light, heat and oxidation. Such technique has been used in the flavoring industry and has a great potential for solving problems related to the use of natural colorants. Thus, this work aimed to apply the microencapsulation technique on natural colorants to enhance their stability and increase their use as food ingredients. The process of encapsulating by spray drying was employed. The colorant chosen was bixin, which is a liposoluble fraction of the pigment urucum (Bixa orellana, L) and the encapsulating agents were maltodextrin, gum arabic and b-cyclodextrin. From these agents, various formulations were obtained by using each agent separately or mixed, followed by stability tests for the micro encapsulated products and for norbixinate (urucum
\end{abstract}


hydrosoluble fraction), used as a control to evaluate the light factor. The microencapsulated products were found to be more stable than the pigment without microencapsulation. Among them, those composed by formulations of gum arabic and maltodextrin mixtures led, in general, to the best performances. The b-cyclodextrin agent was found to be a rather efficient encapsulating agent. Despite its high cost, it is a viable option to increase the stability of natural pigments.

Keywords: Encapsulating agents; Bixin; Colorimetry; Food dyes; Microcapsules.

\section{Resumen}

La microencapsulación es un proceso basado en el confinamiento de especies activas dentro de una cápsula microscópica, que puede brindar protección contra factores lábiles, esta técnica ha sido utilizada en la industria aromatizante y tiene un gran potencial para resolver dificultades relacionadas con el uso de colorantes naturales. El presente trabajo se realizó con el objetivo de aplicar la técnica de microencapsulación en tintes naturales para incrementar su estabilidad y mejorar su uso como ingrediente alimentario. Se utilizó el proceso de encapsulación de "secado por pulverización". El tinte elegido fue bixina, una fracción liposoluble del pigmento de achiote (Bixa orellana, L), y los agentes encapsulantes fueron maltodextrina, goma de acacia y beta-ciclodextrina. De estos tres agentes encapsulantes, se obtuvieron varias formulaciones, usando cada agente por separado o en mezcla. Luego, se realizaron pruebas de estabilidad para los productos microencapsulados y para el norbixinato utilizado como control para evaluar el efecto de la luz. Los productos microencapsulados fueron más estables que los pigmentos sin microencapsulación y, entre estos, los compuestos por una mezcla de acacia y maltodextrina tuvieron, en general, los mejores rendimientos. La beta-ciclodextrina, a su vez, demostró ser un agente encapsulante muy eficaz y, a pesar de su alto costo, aparece como una opción viable para aumentar la estabilidad de los pigmentos naturales.

Palabras clave: Agentes encapsulantes; Bixina; Colorimetría; Tintes alimentarios; Microcápsulas.

\section{Introdução}

Em virtude do crescente interesse pelos corantes naturais, muitos estudos têm sido realizados no que diz respeito a sua química e estabilidade. Embora os corantes artificiais sejam viáveis, econômico e tecnologicamente, eles perdem no mais determinante aspecto, o mercadológico. Atualmente, a sociedade busca a substituição dos produtos artificiais por naturais, por considerá-los mais saudáveis e menos prejudiciais à saúde (Guimarães, 1996; Balem, Alves, Coelho \& Melo, 2017).

Vários fatores aliados a restrições legais reduziram o número de corantes artificias permitidos (Póvoa, 1992). Em contrapartida, viabilizaram o interesse por pesquisas em fontes, extração, estabilidade e rendimento de pigmentos naturais, não só para a aplicação em alimentos, como também na indústria de cosméticos, farmacêutica, têxtil, papel, celulose e, recentemente, tintas e vernizes (Smirnova, Lyskovtseva \& Pletnev, 2021). Todo esse interesse não reside apenas na restrição ao uso dos corantes artificiais, mas também pela potencialidade das características sensoriais, atividades biológicas e antioxidantes dos corantes naturais (Bara \& Vanetti, 1992; Carvalho, 1992; Bonde, 2021). Grande parte destas investigações enfocou o uso de carotenoides, assim como a estabilização de pigmentos mediante tratamentos físicos ou químicos.

A utilização de carotenoides como corantes para alimentos é às vezes dificultada, devido à sensibilidade que esses produtos apresentam a fatores como oxigênio, luz, metais, insolubilidade em água e altos custos quando comparados aos corantes azo. Mesmo assim, as características de corante natural, aliadas às funções fisiológicas que apresentam, coloca-os como os corantes naturais mais utilizados em alimentos (Carvalho, Sarantóploulos, Shirose \& Silva, 1993; Fabri \& Teramoto, 2015).

A bixina, um éster monometílico de um ácido dicarboxilíco denominado norbixina, representa mais de $80 \%$ dos carotenóides presentes no urucum (Coutinho et al., 2016). Devido à sua estrutura molecular, formada por uma série de duplas ligações conjugadas, é instável a luz, oxigênio e temperatura (Najar, Bobbio \& Bobbio, 1988; Silva, Bezerra \& Fernandes, 2018).

As indústrias de corantes naturais têm dado ênfase ao desenvolvimento de formulações que permitam o aumento da estabilidade de tais pigmentos. Uma área particular que tem se mostrado potencialmente promissora no que diz respeito a este assunto é a tecnologia de microencapsulação (Pereira et al., 2018).

Segundo Constant et al. (2020) por meio do processo de microencapsulação é possível melhorar o desempenho dos 
pigmentos naturais quanto a luz, oxigênio e calor quando usados em produtos alimentícios adequados. Isto é verdadeiro para o caso de flavorizantes, que emprega amplamente tal tecnologia.

Tradicionalmente, o método mais comum de encapsulação de ingredientes alimentícios tem sido "spray drying" (Shahadi \& Han, 1993; Jackson \& Lee, 1991; Reineccius, 1989; Ray, Raychaudhuri \& Chakraborty, 2016). O processo é econômico e flexível, com uso de equipamento já disponível e produz partículas de boa qualidade (Risch, 1995; Dziezak, 1988, Ozkan, 2019).

O primeiro passo para a execução da microencapsulação por "spray drying” é a escolha adequada do agente encapsulante a ser empregado. Este deve apresentar propriedades emulsificantes, ter capacidade de formar filmes, mostrar baixa viscosidade a altos níveis de sólidos (menor que 0,5 Pa.s, concentração de sólidos em torno de 45\%), exibir baixa higroscopicidade, ter baixo custo, sabor agradável, ser estável e, principalmente, conferir proteção adequada ao material a ser encapsulado (Risch, 1995; Carmo, Fernandes \& Borges, 2015). Dentre as possíveis substâncias que atendem a estes requisitos, as mais empregadas têm sido os hidrocoloides de grau alimentício goma-arábica, amido modificado e amido hidrolisado (King, 1995; Reineccius, 1988), além de gelatina e proteínas não gelificantes (Dzieak, 1988; Shahadi \& Han, 1993; Vanisk, 2017).

Com base no que foi mencionado, o objetivo deste trabalho foi aplicar a técnica de microencapsulação em corantes naturais, por meio do método do "spray-drying". O corante natural escolhido foi a bixina, que é a fração lipossolúvel do pigmento de urucum, e os agentes encapsulantes foram maltodextrina, goma-arábica e beta-ciclodextrina. Procurou-se comparar os efeitos da concentração de pigmento e o desempenho dos agentes encapsulantes.

\section{Metodologia}

Esta pesquisa foi conduzida segundo procedimentos metodológicos proposto por Pereira, Shitsuka, Perreira \& Shitsuka (2018). O trabalho foi realizado nos laboratórios de Química e Bioquímica de Alimentos do Departamento de Tecnologia de Alimentos da Universidade Federal de Sergipe. Os agentes encapsulantes e o material a ser microencapsulado foram adquiridos comercialmente. $\mathrm{O}$ trabalho foi de natureza analítica quantitativa com a coleta de dados através de grandezas escolhidas como variável de resposta e os resultados analisados estatisticamente.

\subsection{Preparo das microcápsulas}

As matérias-primas empregadas foram goma arábica (Dinâmica), maltodextrina DE-20 (Morex) e beta-ciclodextrina (ACS Científica) como agentes encapsulantes, e suspensão oleosa de urucum com $4 \%$ de bixina (Chr Hansen), como núcleo a ser encapsulado. A Figura 1 mostra o fluxograma do processo de obtenção das microcápsulas de bixina utilizado no presente trabalho.

A partir dos três agentes encapsulantes, goma-arábica, maltodextrina e beta-ciclodextrina, foram efetuadas cinco formulações, empregando-se cada agente tanto separadamente como em mistura. Além de variar o agente encapsulante, alterou-se também a relação encapsulante corante. As formulações empregadas estão descritas no Quadro 1. Na maior parte das formulações foi usado um teor de sólidos de 30\%, de acordo com sugestão da literatura (Reineccius, 1991; Beristain \& Crater, 1994; Sheu \& Rosenberg, 1998). 
Figura 1. Fluxograma geral de processamento.
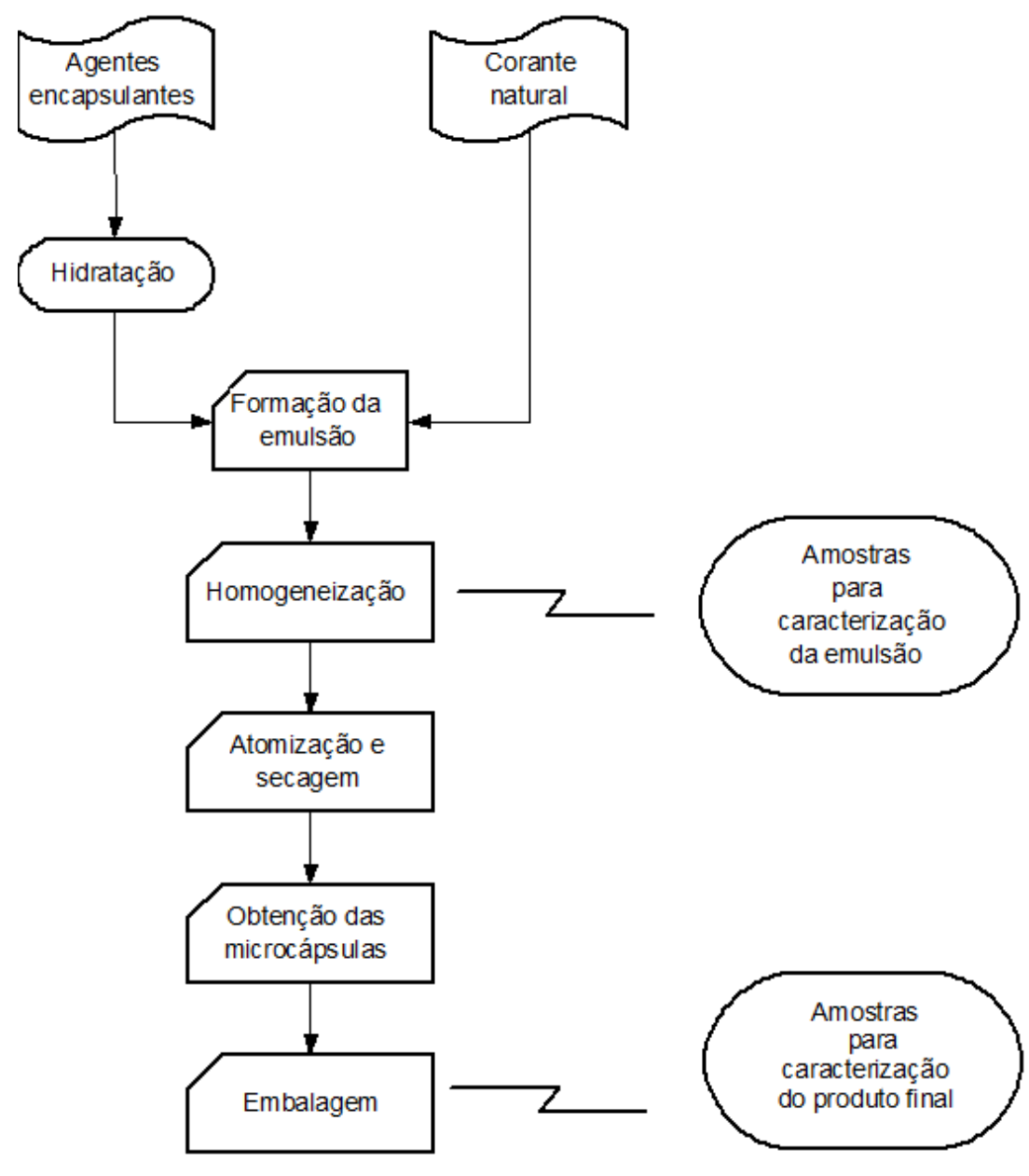

Fonte: Autores (2021).

Quadro 1. Formulações empregadas.

\begin{tabular}{|c|c|c|}
\hline \multicolumn{2}{|c|}{ Formulações } \\
\hline Designação & Concentração dos encapsulantes & Relação corante/encapsulante (p/p) \\
\hline G(1:4) & $30 \%$ Goma-arábica & $1: 4$ \\
\hline G(1:3) & $30 \%$ Goma-arábica & $1: 3$ \\
\hline G(1:5) & $30 \%$ Goma-arábica & $1: 5$ \\
\hline$\beta$ & $10 \%$ Beta-cilclodextrina & $1: 4$ \\
\hline $1 \mathrm{M} 10 \mathrm{G}$ & $10 \%$ Goma-arábica, 20\% maltodextrina DE 10 & $1: 4$ \\
\hline $1 \mathrm{M} 20 \mathrm{G}$ & $10 \%$ Goma-arábica, 20\% maltodextrina DE 20 & $1: 4$ \\
\hline
\end{tabular}

Fonte: Autores (2021).

O procedimento utilizado para o preparo da emulsão foi:

- Hidratação do agente encapsulante por 24 horas a temperatura ambiente, exceto no caso da formulação Beta, em que o encapsulante foi dissolvido no momento da preparação da suspensão com o aquecimento da solução a $65{ }^{\circ} \mathrm{C}$. Para cada formulação foram dissolvidos $300 \mathrm{~g}$ de carboidrato para $1000 \mathrm{~mL}$ de água, exceto para Beta-ciclodextrina empregada pura, sendo dissolvidos $100 \mathrm{~g}$ em $1000 \mathrm{~mL}$ de água;

- $\quad$ Adição do extrato de bixina; e

- Homogeneização da emulsão a 1800 psi em um homogeneizador APV Gaulin. 
As amostras das emulsões foram levadas ao secador tipo "spray dryer" onde procedeu-se a atomização. As condições de secagem foram: vácuo (30 mbar); temperatura do ar de entrada $\left(170 \pm 10{ }^{\circ} \mathrm{C}\right)$; temperatura do ar de saída $\left(90 \pm 5{ }^{\circ} \mathrm{C}\right)$; e pressão manométrica positiva. A quantidade de emulsão atomizada para cada amostra foi em média de $150 \mathrm{~mL}$, e o tempo de secagem variou de 15 a 20 minutos. O processo foi efetuado com três repetições para cada formulação.

\subsection{Caracterização físico-química da emulsão}

Paralelamente ao processo de secagem, foram determinadas algumas das características físico-químicas da emulsão. Dentre as várias propriedades possíveis de serem verificadas, são mostradas a seguir as mais relevantes e que foram executadas:

a) Viscosidade $\rightarrow$ cerca de $150 \mathrm{~mL}$ da emulsão de cada formulação foi separada e foi determinada a sua viscosidade, empregando um reômetro concêntrico, com velocidade de rotação 1190 rpm e temperatura $20^{\circ} \mathrm{C}$.

b) $\mathrm{pH} \rightarrow \mathrm{o} \mathrm{pH}$ foi determinado diretamente em cada emulsão após homogeneização, segundo as Normas Analíticas do Instituto Adolfo Lutz, utilizando um medidor de $\mathrm{pH}$.

c) Qualificação da cor $\rightarrow$ a qualificação da cor foi efetuada pela leitura direta de reflectância das coordenadas "L", "a" e "b", empregando a escala Hunter-Lab em colorímetro.

\subsection{Caracterização do produto microencapsulado.}

Avaliação microscópica $\rightarrow$ o tamanho das microcápsulas foi observado por meio de um microscópio ótico, usando-se objetiva de imersão com aumento de 1.000 vezes. O pó foi diluído a uma concentração $1 \mathrm{~g} / 25 \mathrm{~mL}$ para ser efetuada a observação.

\subsection{Estudo de estabilidade}

Foram realizados testes de estabilidade do produto microencapsulado e da norbixina empregada como testemunha. Os ensaios foram executados conforme metodologia adaptada de Stringheta (1991). As amostras foram solubilizadas em soluçãotampão de Mcllvaine (fosfato dissódico - ácido cítrico) de pH 4. Uma vez preparadas, as soluções com os corantes foram distribuídas em frascos de vidros de capacidade $50 \mathrm{~mL}$, onde recebiam um jato de nitrogênio, para eliminar o oxigênio do espaço livre e, então, eram hermeticamente fechados com tampa de borracha e lacre de alumínio. Parte das amostras era colocada entre lâmpadas fluorescentes de 40W, 2.500 lux, correspondente à luz do dia, mantendo distância de $10 \mathrm{~cm}$ entre si, protegidas de qualquer outra fonte de luz, em ambiente com temperatura controlada de $25 \pm 2{ }^{\circ} \mathrm{C}$. Uma outra fração da amostra foi armazenada ao abrigo de luz, sob a mesma faixa de temperatura. Devido às características do produto em análise (com sólidos em suspensão), não foi efetuada a quantificação direta do pigmento por espectrofotometria, sendo a degradação determinada qualitativamente por colorimetria. Assim sendo, periodicamente, no intervalo de seis horas e seus múltiplos, as amostras eram recolhidas para determinação da cor, em um colorímetro, com leitura direta por reflectância das coordenadas de Hunter "L" (luminosidade), "a" (intensidade de vermelho e verde) e "b" (intensidade de amarelo e azul). O intervalo de leitura entre as amostras expostas a condições diferentes de armazenagem era estabelecido de acordo com o comportamento delas diante de cada situação (para maior degradação, menor o intervalo).

\section{Resultados e discussão}

\subsection{Caraterização físico-química da emulsão}

A Tabela 1 mostra os resultados das características físico-químicas avaliadas para cada emulsão. As formulações que continham apenas o agente encapsulante goma-arábica apresentaram praticamente o mesmo valor de viscosidade. As formulações compostas das misturas goma-arábica/maltodextrina variaram ligeiramente de acordo com a dextrose equivalente 
da maltodextrina. Observou-se que a goma-arábica formou soluções mais viscosas que a maltodextrina e a beta-ciclodextrina respectivamente.

Tabela1. Algumas determinações físico-químicas das emulsões.

\begin{tabular}{c|c|c|c|c|c|c|c}
\hline Amostra & pH & Viscosidade* (mPa.s) & \multicolumn{5}{|c}{ Qualificação da Cor } \\
\cline { 3 - 8 } & & & $\mathrm{L}$ & $\mathrm{a}$ & $\mathrm{b}$ & $\mathrm{c}$ & $\mathrm{h}$ \\
\hline $\mathrm{G} \mathrm{(1:3)}$ & 4,24 & 166,82 & 34,88 & 28,84 & 14,47 & 32,27 & 0,47 \\
\hline G (1:4) & 4,28 & 139,51 & 34,59 & 28,72 & 14,26 & 32,07 & 0,46 \\
\hline G (1:5) & 4,32 & 170,63 & 34,30 & 28,69 & 14,04 & 31,94 & 0,46 \\
\hline 1M10G & 4,47 & 33,41 & 35,46 & 29,28 & 15,05 & 32,92 & 0,47 \\
\hline 1M20G & 4,47 & 36,15 & 35,44 & 29,29 & 15,04 & 32,93 & 0,47 \\
\hline B & 5,18 & 4,57 & 38,60 & 24,71 & 14,62 & 28,71 & 0,53 \\
\hline
\end{tabular}

Legendas: ${ }^{*}$ Velocidade de rotação $190 \mathrm{rpm}$, temperatura $20^{\circ} \mathrm{C} ; \mathrm{G}(1: 3)$ : goma arábica com 1 parte de corante para 4 partes de encapsulante; G (1:4): goma arábica com 1 parte de corante para 3 partes de encapsulante; G (1:5): goma arábica com 1 parte de corante para 5 partes de encapsulante; 1M10G: maltodextrina DE 10 mais goma arábica com 1 parte de corante para 4 partes de encapsulante; 1M20G: maltodextrina DE 20 mais goma arábica com 1 parte de corante para 4 partes de encapsulante; B: betaciclodextrina a $10 \%$ com 1 parte de corante para 4 partes de encapsulante.

Fonte: Autores (2021).

Segundo Reineccius (1989) o valor limite de viscosidade para se proceder a atomização de uma solução é 500,00 $\mathrm{mPa}$.s. De acordo com os resultados, para formulações com o teor de sólidos $30 \%$, o valor máximo de viscosidade observado foi 170,63 mPa.s., inferior ao considerado limite.

Quanto as características colorimétricas as formulações apresentaram tonalidade (h) e saturação de cor (c) semelhantes. A formulação com o agente encapsulante beta-ciclodextrina mostrou-se com maior brilho, menos saturada e apresentando o valor de h maior, ou seja, de acordo com o sólido de Hunter, mais amarelada que as demais. As diferenças observadas entre as amostras foram muito pequenas.

\subsection{Caracterização do produto microencapsulado}

A Tabela 2 mostra o diâmetro das microcápsulas com tamanhos extremos encontrados nas amostras avaliadas. De forma geral, as partículas apresentavam o tamanho intermediário aos valores representados, enquanto a restante exibia tamanho inferior ao limite mínimo.

Tabela 2. Diâmetro das microcápsulas.

\begin{tabular}{cc}
\hline Produto & Diâmetro $(\mu \mathbf{m})$ \\
\hline $\mathrm{G}(1: 3)$ & $5,30-17,58$ \\
$\mathrm{G}(1: 4)$ & $5,30-15,98$ \\
$\mathrm{G}(1: 5)$ & $5,08-12,17$ \\
$1 \mathrm{M} 10 \mathrm{G}$ & $4,90-8,56$ \\
$1 \mathrm{M} 20 \mathrm{G}$ & $4,45-10,66$ \\
$\mathrm{~B}$ & - \\
\hline
\end{tabular}

Legendas: G (1:3): goma arábica com 1 parte de corante para 4 partes de encapsulante; G (1:4): goma arábica com 1 parte de corante para 3 partes de encapsulante; G (1:5): goma arábica com 1 parte de corante para 5 partes de encapsulante; $1 \mathrm{M} 10 \mathrm{G}$ : maltodextrina DE 10 mais goma arábica com 1 parte de corante para 4 partes de encapsulante; 1M20G: maltodextrina DE 20 mais goma arábica com 1 parte de corante para 4 partes de encapsulante; B: betaciclodextrina a $10 \%$ com 1 parte de corante para 4 partes de encapsulante.

Fonte: Autores (2021)

As amostras, a não ser pela formulação Beta cuja encapsulação se dá em nível molecular, apresentaram em comum a tendência de formação de cápsulas com formato preferencialmente esférico e com diâmetros bastante variados. 


\subsection{Estudo de estabilidade}

Constant (1999) observou que, para fins de verificação da degradação do pigmento empregado neste trabalho, a coordenada colorimétrica "a" é a mais representativa, sendo por esta razão empregada para análise dos resultados. Os dados obtidos nas análises colorimétricas foram analisados estatisticamente e os modelos de regressão foram ajustados por meio do programa estatístico SAS versão 6.12 (Statistical Analysis System - SAS Institute Inc., Cary, NC, USA).

As Figuras 2 e 3 apresentam os gráficos referente ao comportamento da coordenada a no decorrer do tempo respectivamente em ausência e presença de luz.

Figura 2. Comportamento de "a" (intensidade de vermelho e verde) ao longo do tempo, para as diferentes formulações em ausência de luz, em pH 4 , à temperatura de $25 \pm 2{ }^{\circ} \mathrm{C}$.

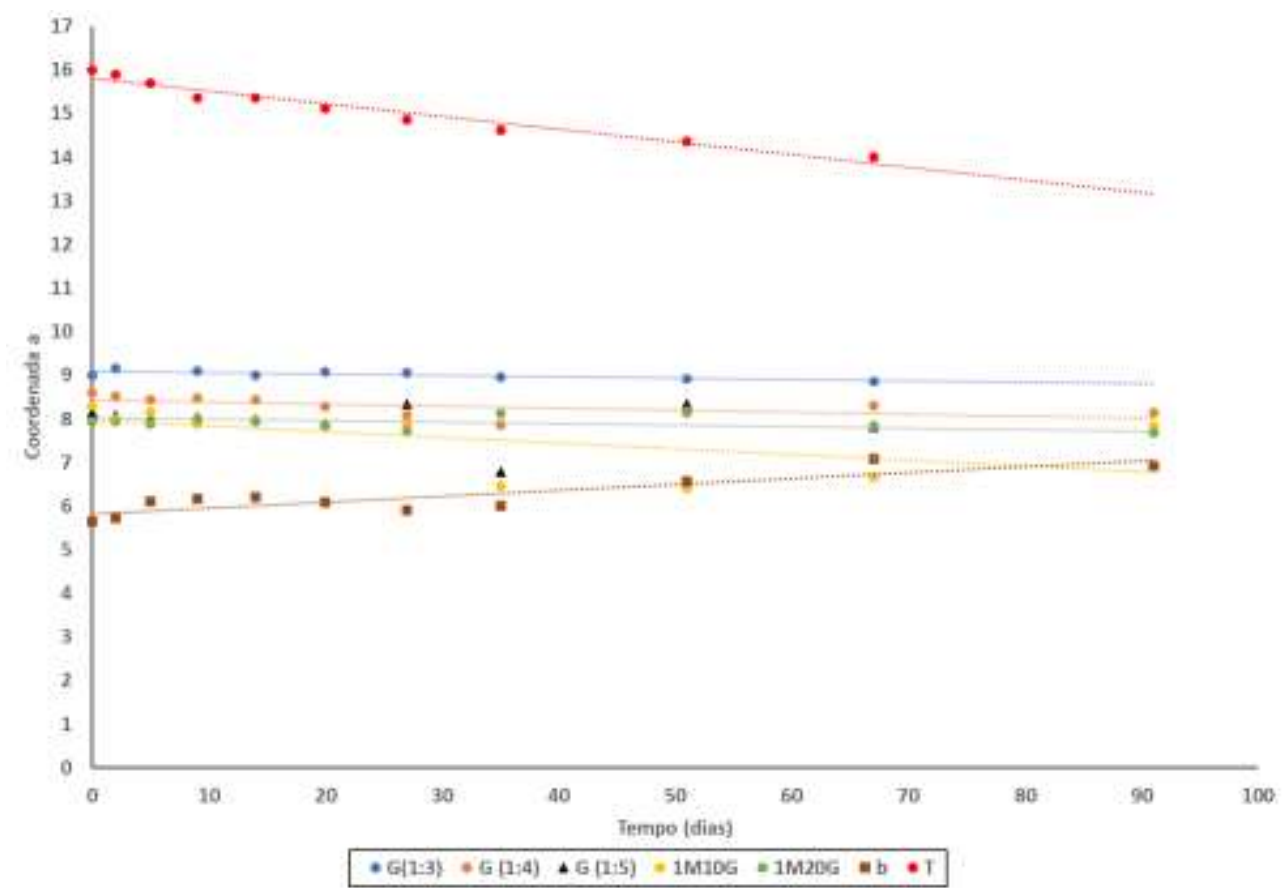

Legendas: G (1:3): goma arábica com 1 parte de corante para 4 partes de encapsulante; G (1:4): goma arábica com 1 parte de corante para 3 partes de encapsulante; G (1:5): goma arábica com 1 parte de corante para 5 partes de encapsulante; 1M10G: maltodextrina DE 10 mais goma arábica com 1 parte de corante para 4 partes de encapsulante; 1M20G: maltodextrina DE 20 mais goma arábica com 1 parte de corante para 4 partes de encapsulante; b: betaciclodextrina a $10 \%$ com 1 parte de corante para 4 partes de encapsulante; T: norbixina usada como testemunha.

Fonte: Autores (2021). 
Figura 3. Comportamento de "a" (intensidade de vermelho e verde) ao longo do tempo, para as diferentes formulações em presença de luz, em $\mathrm{pH} 4$, à temperatura de $25 \pm 2{ }^{\circ} \mathrm{C}$.

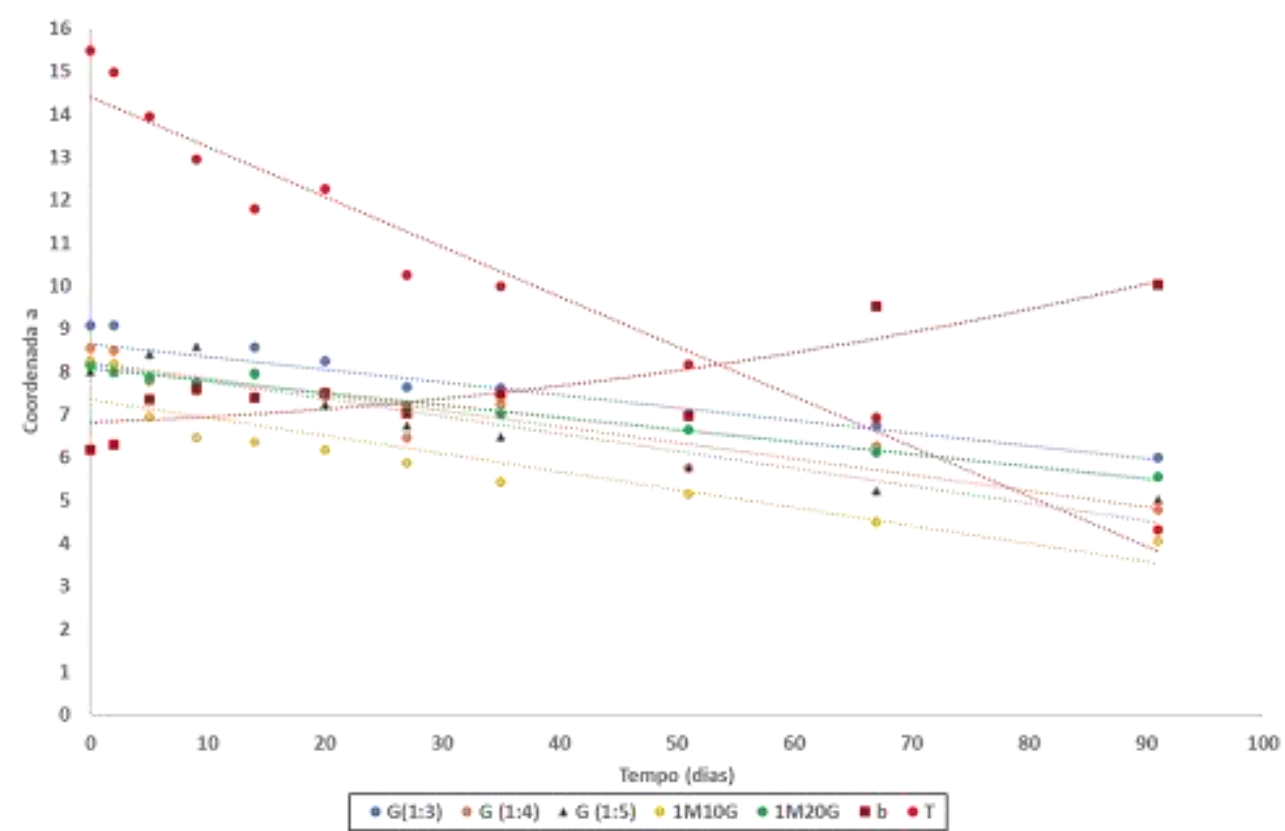

Legendas: G (1:3): goma arábica com 1 parte de corante para 4 partes de encapsulante; G (1:4): goma arábica com 1 parte de corante para 3 partes de encapsulante; G (1:5): goma arábica com 1 parte de corante para 5 partes de encapsulante; 1M10G: maltodextrina DE 10 mais goma arábica com 1 parte de corante para 4 partes de encapsulante; 1M20G: maltodextrina DE 20 mais goma arábica com 1 parte de corante para 4 partes de encapsulante; b: betaciclodextrina a $10 \%$ com 1 parte de corante para 4 partes de encapsulante; T: norbixina usada como testemunha.

Fonte: Autores (2021).

A não ser pela formulação Beta, que teve comportamento particular em cada situação, as demais amostras comportaram-se de maneira similar, não apresentando sensível variação do valor "a" no escuro e declínio do mesmo em situações de incidência de luz. Em ambas as situações, a testemunha apresentou modificações bem mais acentuadas.

Uma vez que o valor "a' quantifica a intensidade de vermelho, este fenômeno era esperado, visto que em processo de degradação os componentes vermelho/alaranjado que constituem os pigmentos do urucum transformam-se nos chamados compostos amarelos. Estes compostos são formados pela mistura de uma série de substâncias como isômeros da bixina, produtos com pesos moleculares inferiores provenientes da quebra da cadeia carbônica do pigmento ou anéis aromáticos com o m-xileno (McKeown \& Mark, 1962; Scotter, Thorpe, Reynolds, Wilson \& Strutt, 1994). Portanto, o valor de "a" deve diminuir com o tempo se estiver ocorrendo alguma degradação do produto avaliado.

A Figura 4 compara os resultados obtidos para as formulações testadas com diferentes relações corante/agente encapsulante (formulações com a goma arábica como único agente encapsulante). É possível verificar que aquela cuja razão foi de 1:3 teve melhor desempenho, ou seja, manteve-se mais estável ao longo do tempo. Uma vez que esta formulação apresentava a maior concentração de corante, tal resultado é interessante por mostrar que, em se tratando de pigmentos, o comportamento parece não ser o mesmo verificado para o caso de flavorizantes em que o limite máximo usualmente empregado de material ativo é $25 \%$ (Risch, 1995). 
Figura 4. Comportamento de "a" (intensidade de vermelho e verde) ao longo do tempo, para formulações testadas com diferentes relações corante/agente encapsulante, em presença de luz, em pH 4 , à temperatura de $25 \pm 2{ }^{\circ} \mathrm{C}$.

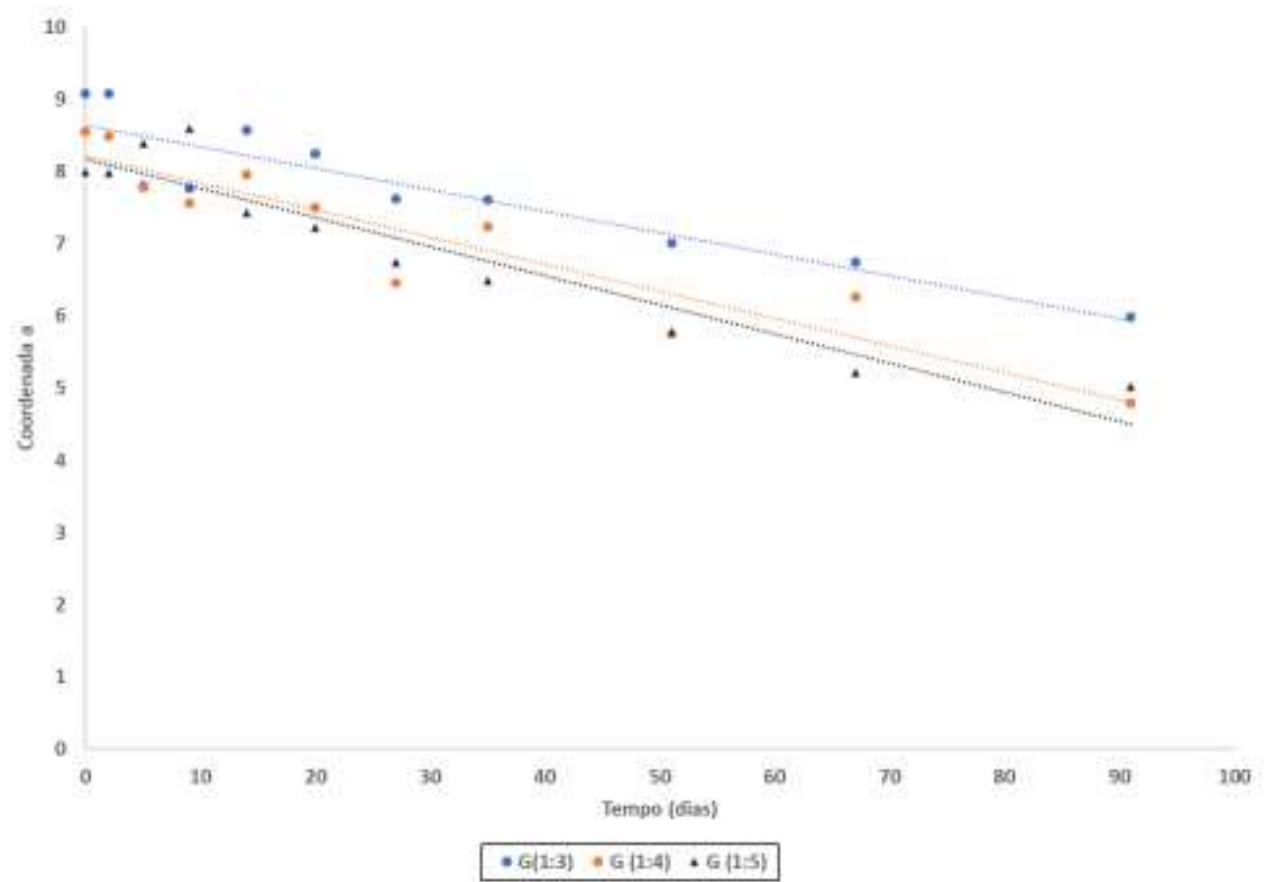

Legendas: G (1:3): goma arábica com 1 parte de corante para 4 partes de encapsulante; $\mathrm{G}$ (1:4): goma arábica com 1 parte de corante para 3 partes de encapsulante; G (1:5): goma arábica com 1 parte de corante para 5 partes de encapsulante.

Fonte: Autores (2021).

Entre as maltodextrinas testadas, aquela com dextrose equivalente maior (DE 20) foi mais eficaz. Tal resultado é mostrado na Figura 5.

Figura 5. Comportamento de "a" (intensidade de vermelho e verde) ao longo do tempo, para formulações testadas com maltodextrina com diferentes $\mathrm{DE}$, em presença de luz, em $\mathrm{pH} 4$, à temperatura de $25 \pm 2{ }^{\circ} \mathrm{C}$.

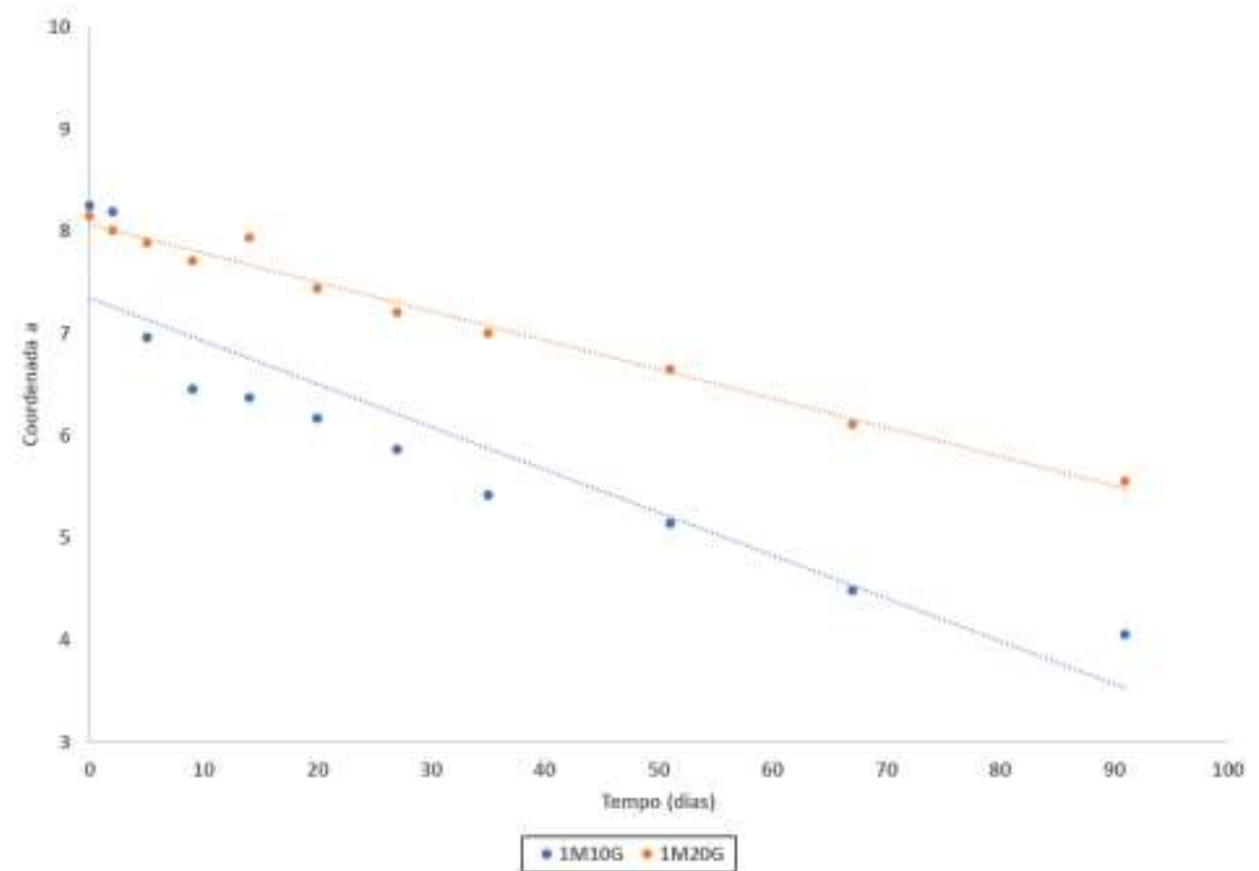

Legendas: 1M10G: maltodextrina DE 10 mais goma arábica com 1 parte de corante para 4 partes de encapsulante; 1M20G: maltodextrina DE 20 mais goma arábica com 1 parte de corante para 4 partes de encapsulante.

Fonte: Autores (2021). 
A Figura 6 mostra os resultados para as formulações onde foram empregados distintos agentes encapsulante e a mesma proporção corante/encapsulante.

Figura 6. Comportamento de "a" (intensidade de vermelho e verde) ao longo do tempo, para as diferentes formulações em presença de luz, em $\mathrm{pH} 4$, à temperatura de $25 \pm 2{ }^{\circ} \mathrm{C}$.

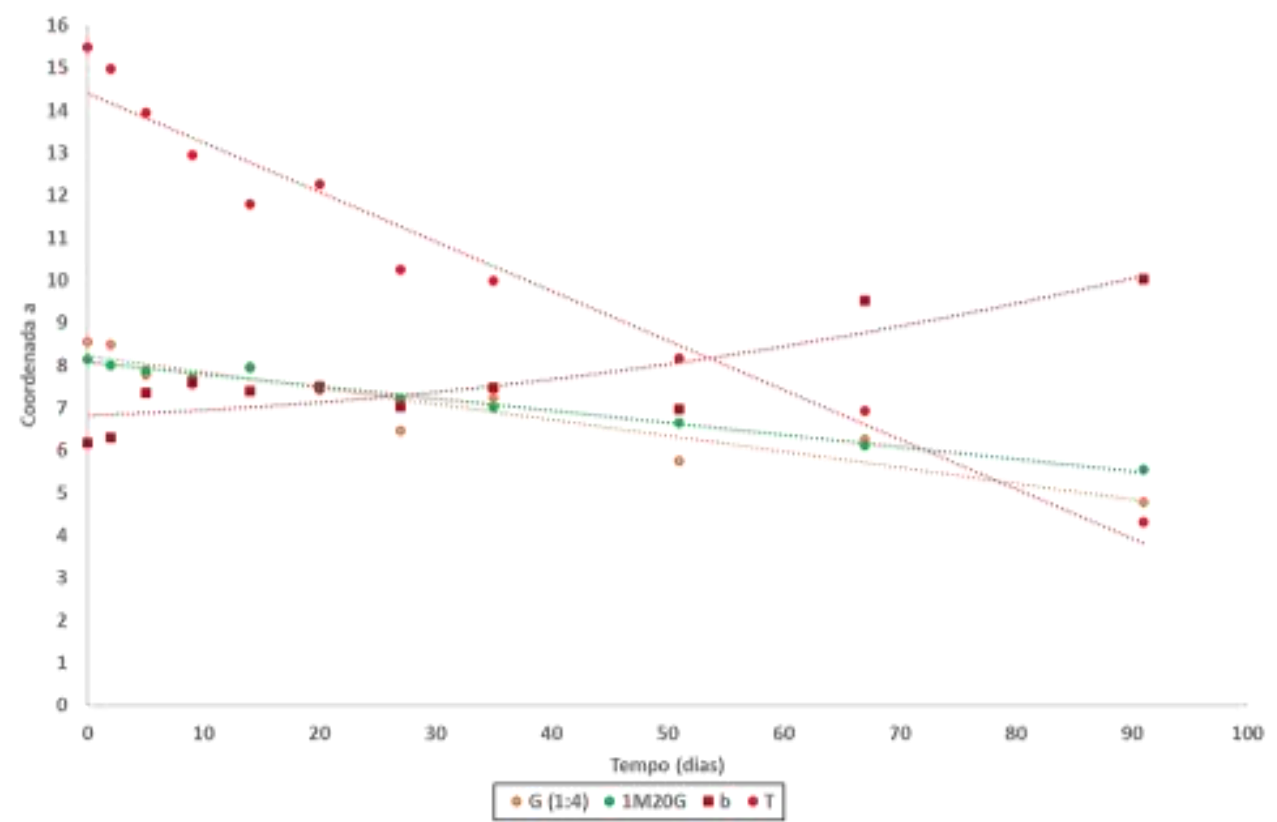

Legendas: G (1:4): goma arábica com 1 parte de corante para 3 partes de encapsulante; 1M20G: maltodextrina DE 20 mais goma arábica com 1 parte de corante para 4 partes de encapsulante; b: betaciclodextrina a $10 \%$ com 1 parte de corante para 4 partes de encapsulante; T: norbixina usada como testemunha. Fonte: Autores (2021).

Quanto aos agentes encapsulantes empregados, a goma-arábica mostrou ser um excelente agente emulsificante, mas a mistura goma/maltodextrina conferiu maior proteção ao produto promovendo menores mudanças na coordenada colorimétrica avaliada. Um fato interessante a ser observado é que o esperado em uma situação de degradação do pigmento do urucum é que o valor de "a" diminua com o tempo, seja de forma acentuada, como no caso da testemunha, seja suavemente, como no produto microencapsulado. Isto não é observado para o caso da formulação Beta. Ao contrário, seu valor de "a" aumenta com o tempo, tanto na luz quanto no escuro.

Tal fato pode ser explicado pela possível aproximação das moléculas do complexo beta-ciclodextrina/bixina, formando aglomerados de forma a se ter uma percepção maior da cor vermelha. O comportamento semelhante observado nas situações de incidência e ausência de luz sugere que a beta-ciclodextrina tenha capacidade de formar complexos com o pigmento muito estáveis, sendo pouco afetados pela luz, que é considerada o agente mais destrutivo para o corante de urucum. No entanto, a baixa solubilidade do complexo formado torna-o pouco viável para emprego em produtos líquidos.

\section{Conclusão}

O processo de encapsulação por "spray drying" é simples de ser conduzido, no entanto pequenas alterações nas condições de homogeneização e secagem provocam variações na estrutura das microcápsulas obtidas. De forma geral, as partículas apresentaram formato esférico, exceto para a beta-ciclodextrina, cuja encapsulação se dá em nível molecular.

Dentre os parâmetros usados para avaliar a estabilidade das amostras, o valor "a" (intensidade de vermelho) demonstrou ser o mais representativo.

O corante microencapsulado mostrou maior estabilidade diante do fator luz que o corante não microencapsulado 
usado como testemunha neste trabalho. O processo de microencapsulação é uma técnica que, uma vez usada em pigmentos naturais, pode oferecer proteção e torná-los mais estáveis.

Entre as formulações com diferentes relações corante/encapsulante, aquela cuja relação foi 1:3 apresentou melhor desempenho. Quanto aos agentes encapsulantes, a mistura goma-arábica/ maltodextrina conferiu maior proteção ao produto. Para as diferentes maltodextrinas testadas, aquela com dextrose equivalente (DE) mais elevada foi mais eficaz.

A beta-ciclodextrina gerou complexos muito estáveis, não sendo afetado sensivelmente pela luz, que é considerada o agente mais destrutivo para o pigmento do urucum (Bixa orelleana). Apesar de a solubilidade em água da bixina ser melhorada, o complexo bixina/beta-ciclodextrina apresentou-se pouco solúvel.

Futuros trabalhos podem aplicar o corante de urucum microencapsulados em diferentes sistemas alimentícios estudando para cada caso a estabilidade e manutenção da coloração durante a vida útil dos alimentos.

\section{Referências}

Balem, T. A., Alves, E. O., Coelho, J. C., \& Mello, A. L. P. (2017). Food transformations in modern society: the colonization of natural food by industrial food. Revista Espacios, 38(47), 5-18.

Bara, M. T. F., \& Vanetti, M. C. D. (1992) Atividade microbiana de corantes naturais sobre microrganismos patogênicos veiculados por alimentos. Revista Brasileira de Corantes Naturais, 1(1), 194-200.

Beristiain, C. I., \& Carter, E. J. V. (1994). Utilization of mesquite (Prosopis juliflora) gum as stabilizing agente for spray dried encapsulated orange peel oil. Dry Technology, 12(7). 1727-1733. 10.1080/07373939408962195

Bonde. Entenda os benefícios dos corantes naturais para a saúde. (2021). https://www.bonde.com.br/saude/nutricao/entenda-os-beneficios-dos-corantesnaturais-para-a-saude-247325.html.

Carmo, E. L., Fernandes, R. V. B., \& Borges, S. V. (2015). Microencapsulação por spray drying, novos biopolímeros e aplicações na tecnologia de alimentos. Journal of Chemical Engineering and Chemistry. 1(2), 30-44. 10.18540/jcecvl1iss2pp30-44

Carvalho, P. R. N., Sarantóploulos, G. L., Shirose, I., \& Silva, M. G. (1993). Estudo de vida-de-prateleira do corante (bixina) extraído das sementes de urucum (Bixa orellana, L). Coletânea do ITAL, 23(1), 98-104.

Carvalho, P. R. N. (1992). Corantes de urucum hidrossolúveis. Revista Brasileira de Corantes Naturais, 1(1), $244-245$.

Constant, P. B. L. (1999). Microencapsulamento de bixina: agentes encapsulantes, avaliação da qualidade e aplicações. (Dissertação de Mestrado) Universidade Federal de Viçosa, Viçosa, MG.

Constant, P. B. L., Melo, T. S., Pimentel, A. A., Araujo, R. R. S., Fanchiotti, F. E., Silva, A. G., \& Santos, J. A. B. (2020) Application of microencapsulated bixin in food systems. Brazilian Journal of Development. 6(7). 47604-47617. 10.34117/bjdv6n7-411

Coutinho, M. A. S., Lima, E. C., Guimarães, M. M. M., Matos, A. Z. G., Alves, A. L., Silva, R. B., \& Viana, V. G. F. (2016). Determinação da eficácia de diferentes solventes orgânicos na dissolução dos corantes naturais bixina e norbixina. Revista UniVap. 22(40). 10.18066/revistaunivapv22i40.934

Dziezak, J. D. (1988). Microencapsulation and encapsulated ingredients. Food Technology, 42(4), 136-148.

Fabri, E. G.; \& Teramoto, J. R. S. (2015). Urucum: fonte de corantes naturais. Horticultura Brasileira, 33(1). 10.1590/S0102-053620150000100023

Guimarães, I. S. S. (1996). Corantes naturais: sucedâneos dos artificiais. Revista Brasileira de Corantes Naturais, 2(1), 98-110.

Instituto Adolfo Lutz. Normas analíticas do Instituto adolfo Lutz. (31a ed.).

Jackson, L. S., \& Lee, K. (1991). Microencapsulation and the food industry. Lebensmittel-Wissenschaft und -Technologie, 24(4), $289-297$.

King, A. H. (1995). Encapsulation of food ingredients: a review of available technology, focusing hidrocolloids. In: Risch, S. J., Reineccius, G. A., Encapsulation and controlled release of food ingredients. ACS. (ACS Symposium Series 590).

McKeown, G. G., \& Mark, E. (1962). The composition of oil-soluble annatto food colors. Journal of the A.O.A.C., 45(3), $761-766$.

Najar, S. V., Bobbio, F. O., \& Bobbio, P. A. (1988). Effects of light, air, anti-oxidants and pro-oxidants on anntto extracts (bixa orellana). Food Chemistry, 29(4), 283-289.

Ozkan, G., Franco, P., Marco, I., Xiao, J., \& Capanoglu, E. (2019). A review of microencapsulation methods for food antioxidants: Principles, advantages, drawbacks and applications. Food Chemistry. 272, 494-506. 10.1016/j.foodchem.2018.07.205

Pereira, K. C., Ferreira, D. C. M., Alvarenga, G. F., Pereira, M. S. S., Barcelos, M. C. S., \& Costa, J. M. G. (2018). Microencapsulation and release controlled by the diffusion of food ingredients produced by spray drying: a review. Brazilian Journal of Food Technology. 21, 1-9. 10.1590/1981-6723.08317 
Research, Society and Development, v. 10, n. 9, e51910918179, 2021

(CC BY 4.0) | ISSN 2525-3409 | DOI: http://dx.doi.org/10.33448/rsd-v10i9.18179

Pereira A. S., Shitsuka, D. M., Perreira, F. J., \& Shitsuka, R. (2018). Metodologia da pesquisa científica. UFSM. Disponível em: https://repositorio.ufsm.br/bitstream/handle/1/15824/Lic_Computacao_Metodologia-Pesquisa-Cientifica.pdf?sequence=1

Póvoa, M. E. B. (1992). Extração do corantes do urucum (Bixa orellanna L) com diversos solventes. Revista Brasileira de Corantes Naturais, 1(1), 153-157.

Ray, S.; Raychaudhuri, U.; \& Chakraborty, R. (2016). An overview of encapsulation ofactive compoundsused in food products by drying technology. Food Bioscience, 13, 76-83. 10.1016/j.fbio.2015.12.009

Reineccius, G. A. (1991). Carbohydrates for flavor encapsulation. Food Technology, 45(3), 144-149.

Reineccius, G. A. Flavor encapsulation. Food Rewiews International, 5(2), 147-176, 1989.

Reineccius, G. A. (1988). Spray-drying of food flavors In: Risch, S. J., Reineccius, G. A., Flavor Encapsulation. ACS, 55-66. (ACS Symposium Series 370).

Risch, S. J. (1995). Encapsulation: overview of uses and techniques. In: RISCH, S. J., REINECCIUS, G. A., Encapsulation and controlled release of food ingredients. (ACS Symposium Series 590). 10.1021/bk-1995-0590

Scotter, M. J., Thorpe, S. A., Reynolds, S. L., Wilson, L. A., \& Strutt, P. R. (1994). Characterization of the principal colouring components of annatto using higt performance liquid chromatography with photodiode-array detectio. Food Additives and Contaminants, 11(3), 301-315. 10.1080/02652039409374229

Shahidi, F., \& Han, X. (1993). Encapsulation of food ingredients. Critical Reviews in food Science and Nutrition, 36(6), 501-547. $10.1080 / 10408399309527645$

Sheu, T-Y., \& Rosenberg, M. (1998). Microestruture of microcapsules consisting of whey proteins and carbohydrates. Journal of Food Science, 63(3), 491494. $10.1111 /$ j.1365-2621.1998tb15770

Silva, F. A., Bezerra, A. M. C., \& Fernandes, P. R. D. (2018). Testes fitoquímicos em extratos orgânicos de Bixa orellana L (Urucum). Holos. 34(2), 484-498. 10.15628/holos.2018.6929

Smirnova, S. V., Lyskovtseva, K. A., \& Pletnev, I. V. (2021). Extraction and determination of synthetic food dyes using tetraalkylammonium based liquidliquid extraction. Microchemical Journal. 162. 105833-105842. :10.1016/j.microc.2020.105833

Stringheta, P. C (1991). Identificação da estrutura e estudo da estabilidade das antocianinas extraídas da inflorescência de capim gordura (Mellinis minutiflora, Pal de Beauv). (Tese de Doutorado), Universidade Estadual de Campinas, Campinas, SP.

Vaniski, R., Corti, D., \& Drunkler, D. A. (2017). Técnicas e materiais empregados na microencapsulação de probióticos. Brazilian Journal of Food Research. 8(1), 156-184. 10.3895/rebrapav8n1.3651. 